\begin{tabular}{|c|l|}
\hline Title & A ssembling of nanoparticles using ice crystals \\
\hline Author(s) & Mukai, Shin R.; Mitani, Kazuhiro; Murata, Shinya; Nishihara, Hirotomo; Tamon, Hajime \\
\hline Citation & $\begin{array}{l}\text { Materials Chemistry and Physics, 123(2-3), 347-350 } \\
\text { https://doi.org/10.1016/.matchemphys.2010.05.025 }\end{array}$ \\
\hline Issue Date & 2010-10-01 \\
\hline Doc URL & http://hdl.handle.net/2115/43933 \\
\hline Type & article (author version) \\
\hline File Information & MCP123-2-3_347-350.pdf \\
\hline
\end{tabular}

Instructions for use 


\title{
Assembling of Nanoparticles Using Ice Crystals
}

\author{
${ }^{* 1}$ Shin R. Mukai, ${ }^{* 2}$ Kazuhiro Mitani, ${ }^{* 1}$ Shinya Murata, ${ }^{* 3}$ Hirotomo Nishihara and ${ }^{* 2}$ Hajime Tamon \\ ${ }^{* 1}$ Graduate School of Engineering, Hokkaido University, Sapporo, Japan \\ ${ }^{* 2}$ Graduate School of Engineering, Kyoto University, Kyoto, Japan \\ ${ }^{*}$ Institute of Multidisciplinary Research for Advanced Materials, Tohoku University, Sendai Japan
}

\begin{abstract}
This article introduces a method to assemble nanoparticles into the form of fibers and monolithic microhoneycombs using unidirectional freezing. The nanoparticles used were of $\mathrm{TiO}_{2}$, high $\mathrm{SiO}_{2}$ Y-type zeolite, and graphite. A silica hydrogel was used as the binder, and an array of needle-shaped ice crystals formed during the unidirectional freezing of the particle-hydrogel complex was used as the template. It was found that such particle-hydrogel complexes can be easily molded into the form of fibers and monolithic microhoneycombs like hydrogels which do not include particles. The morphology can be changed by adjusting the firmness of the silica hydrogel. The strength of the microhoneycombs were found to decrease with the inclusion of nanoparticles, but could be recovered through calcination. The fibers and monolithic microhoneycombs are expected to have the combined functions of the particles and binder included within them. They are also equipped with a network of nanopores and macropores which reach to the surface of the nanoparticles, therefore various fluids can gain access to the surface of the nanoparticles quite easily. Due to this high accessibility, it is expected that the included nanoparticles can show their maximum performances.
\end{abstract}

Keywords: microporous materials, sol-gel growth, adsorption, microstructure

\section{Introduction}

Various inorganic metal oxides having unique functions can be synthesized through sol-gel transition. Such metal oxides are generally synthesized in a particle or powder form, but they can also be synthesized in the form of fibers by freezing their parent hydrogels "unidirectionally."1-3 Fibers obtained through this method are generally porous, which indicates the possibility of using them as adsorbents and/or catalysts. ${ }^{4}$ Moreover, the diameters of the fibers, as well as the sizes of the pores within them, can be controlled independently by simply adjusting preparation conditions.

Unidirectional freezing was originally used to obtain long and strong fibers, so thoroughly aged hydrogels were typically used as the precursor. However, we found that by applying this method to hydrosols or freshly gelled soft hydrogels, gels with unique morphologies, such as lamellar sheets, flat fibers and microhoneycombs, can also be obtained. ${ }^{5,6}$ We also found that the morphology which appears during unidirectional freezing can be controlled by adjusting the firmness of the precursor sol or gel. ${ }^{7}$ As this method practically uses ice crystals as the template, we named it the "ice templating method." 
Microhoneycombs obtained through the ice templating method have straight channels, which sizes can be adjusted roughly in the range of about 5 to about 100 micrometers by changing freezing conditions. The thicknesses of the walls which form the channels are typically about 1 micrometer. This means that the pressure drop which occurs when fluids are passed through them will be extremely low even though the diffusion path lengths within the porous walls are extremely short. This feature cannot be attained in conventional columns which are packed with particles. Therefore, this method is expected to provide porous materials with an ideal morphology which can be used in various systems. However, the applicability of this method is limited to materials which can be synthesized through the sol-gel method.

In this work, we attempted to widen the range of applicability of the ice templating method. We applied the ice templating method to $\mathrm{SiO}_{2}$ hydrogels including various nanoparticles which have different surface properties and which possess different functions. $\mathrm{SiO}_{2}$ is expected to act as the binder and also to provide a large surface area to the resulting material. This modified ice templating method is expected to open up a route to synthesize materials not only with unique morphologies, but also with multiple functions.

\section{Experimental}

\subsection{Materials}

Sodium silicate solutions from Wako Pure Chemical Industries were used for $\mathrm{SiO}_{2}$ hydrogel preparation. $\mathrm{TiO}_{2}$ nanoparticles (P25, average particle size $21 \mathrm{~nm}$ ) manufactured by Degussa, high $\mathrm{SiO}_{2}$ Y-type zeolite particles (HSZ-390, average particle size $300 \mathrm{~nm}$ ) manufactured by Tosoh and graphite particles (particle size under $5 \mu \mathrm{m}$ ) distributed by Wako Pure Chemical Industries were used as the particles. Ion exchange resins manufactured by Rohm and Haas and distributed by Organo Corporation (Amberlite IR120B H) were used to adjust the pH of the $\mathrm{SiO}_{2}$ hydrogel precursor.

\subsection{Material Synthesis}

Commercial sodium silicate solutions were diluted with ion-exchanged and distilled water, and the $\mathrm{SiO}_{2}$ concentration in them were adjusted to $1.9 \mathrm{~mol} / \mathrm{L}$. This $\mathrm{SiO}_{2}$ concentration was adopted as it gives a firm hydrogel at a moderate gelation time. Next, ion-exchange resin particles were immersed into the solutions, and the resins were immediately filtered out when the $\mathrm{pH}$ of the solutions reached about 2.5. To these solutions, various particles were added. The particles used in this work were high $\mathrm{SiO}_{2}$ Y-type zeolite particles, a material which has the same composition as the binder, $\mathrm{TiO}_{2}$ nanoparticles, a hydrophilic material, and graphite particles, a hydrophobic material. The particles also have unique functions, high $\mathrm{SiO}_{2}$ Y-type zeolite particles are used as catalysts in various petrochemical processes, $\mathrm{TiO}_{2}$ nanoparticles are used as photocatalysts, and graphite particles are used as a chemically stable and electric conducting material. They will be respectively denoted as p25, HSZ and GRA, hereafter. First, the particles were dispersed in the solutions using an ultrasonic homogenizer. The amount of particles was adjusted so that the particle/ $\mathrm{SiO}_{2}$ ratio in the resulting material would be in the range 0.2 to 2.0 g-particle $/ \mathrm{g}-\mathrm{SiO}_{2}$. The resulting slurries were poured into polypropylene tubes (i.d.: $10 \mathrm{~mm}, L: 120 \mathrm{~mm}$ ) and were aged for different periods. Next the tubes were dipped at a constant rate of $8 \mathrm{~cm} / \mathrm{h}$ into a cold bath maintained at $77 \mathrm{~K}$. After the tubes were completely frozen, they were taken out from the bath and thawed at 323 
K. Then the water in the tubes was exchanged with 2-methyl-2-propanol to reduce the surface tension caused by the solvent during drying to protect porosity, and the samples were freeze-dried by maintaining them under vacuum at $263 \mathrm{~K}$.

\subsection{Characterization}

The morphology of the samples was directly observed using a scanning electron microscope (SEM, JEOL Ltd.; JSM-6340FS). The samples were analyzed using an X-ray diffractometer (XRD, Rigaku Co. Ltd.; RINT2100 $(\mathrm{CuK} \alpha))$ to check whether the included particles maintained their structures. The porous properties of the samples were evaluated through nitrogen adsorption experiments. First, nitrogen adsorption isotherms of the samples were measured at $77 \mathrm{~K}$ using an adsorption apparatus (BEL Japan Inc.; Belsorp 28). The samples were heat treated at $523 \mathrm{~K}$ for $4 \mathrm{~h}$ in vacuum prior to adsorption measurements. Next, BET surface areas and mesopore volumes of the samples were calculated from the obtained isotherms. The compression strengths of honeycomb type samples were also measured using a load cell (A\&D Company, Ltd. max. $3 \mathrm{~kg}$ ). Both the diameter and length of the specimens used in compression strength measurements were $10 \mathrm{~mm}$. Finally, the pressure drop which occurs when air was passed through typical microhoneycombs was measured.

\section{Results and Discussion}

It was anticipated that the inclusion of nanoparticles will alter the gelation behavior of $\mathrm{SiO}_{2}$ which was used as the binder in this work. This may become a crucial issue, especially when the gelation time becomes extremely short. However, regardless of the type of nanoparticle, the gelation time of $\mathrm{SiO}_{2}$ including nanoparticles was about $2.5 \mathrm{~h}$, the same as $\mathrm{SiO}_{2}$ not including any particles.

In the original ice templating method, it was found that the morphology which appears during freezing depends on the firmness of the precursor hydrogel. ${ }^{7}$ A microhoneycomb structure appears when a fairly soft hydrogel is used, and a firm hydrogel gives a fibrous structure. The firmness of a hydrogel can be easily controlled by adjusting the time allowed for it to age. Therefore, in this work we adjusted the aging time of the particle- $\mathrm{SiO}_{2}$ mixtures prior to freezing. The aging time was adjusted to $2 \mathrm{~h}$, when the synthesis of microhoneycombs was intended, whereas when fibers were desired, the aging time was adjusted to $50 \mathrm{~h}$.

In the synthesis of microhoneycombs and fibers using the ice templating method, the ice templates must grow under a steady state, in order to obtain products with a uniform structure. Therefore it was also anticipated that the nanoparticles used in this work may disturb the growth of ice templates. However, it was confirmed that monolithic microhoneycombs and fibers of $\mathrm{SiO}_{2}$ including p25, HSZ and GRA particles can be synthesized using the modified ice templating method. Figure 1 shows SEM images of typical samples obtained in this work. The particle $/ \mathrm{SiO}_{2}$ ratio is 0.6 g-particle $/ \mathrm{g}-\mathrm{SiO}_{2}$ for microhoneycomb samples, and that for fiber samples is 0.2 g-particle/g- $\mathrm{SiO}_{2}$. As expected, the morphology which appears after freezing could be controlled by adjusting the time allowed for the precursor to age prior to freezing.

There was a noticeable limit in the amount of nanoparticle inclusion in order to obtain self-standing samples. The limit depended on the type of nanoparticle included. Self-standing microhoneycombs with a high particle $/ \mathrm{SiO}_{2}$ ratio of $2.0 \mathrm{~g}$-particle/g- $\mathrm{SiO}_{2}$ could be obtained when $\mathrm{HSZ}$ particles was used. This is probably due to the fact that the composition of $\mathrm{HSZ}$ and the $\mathrm{SiO}_{2}$ binder are quite similar. On the other hand, it was difficult to 
obtain microhoneycombs including p25 or GRA particles with particle/SiO $\mathrm{S}_{2}$ ratios over $1.0 \mathrm{~g}$-particle/g-SiO ${ }_{2}$. Long fibers including nanoparticles could not be obtained when the particle/ $/ \mathrm{SiO}_{2}$ ratio exceeded 0.2 g-particle/g- $\mathrm{SiO}_{2}$. As porous fibers can be obtained through other methods, hereafter we will focus on the unique monolithic microhoneycomb samples.

The thickness of the walls of the obtained microhoneycombs seemed to depend on the sizes of the included particles which increase in the order p25, HSZ and GRA. Although the channels of the microhoneycombs were not as straight and as regularly ordered as those in the microhoneycombs obtained through the original ice templating method, it was confirmed through ink penetration tests that the channels run from one side to the other side of the honeycomb.

Figure 2 shows the XRD patterns of typical samples. All patterns exhibit a broad feature in the low angle zone, which is due to the $\mathrm{SiO}_{2}$ binder. In addition, reflections corresponding to the crystal structure of the included particles can be clearly observed, which indicates that the included particles maintained their nanostructure. Therefore, the particles are assumed to maintain their unique functions, such as catalysis and adsorption abilities, after processing.

The porous properties of the samples were evaluated through nitrogen adsorption experiments. The nitrogen adsorption isotherms of typical samples are shown in Fig. 3. From the shape of the isotherms, the existence of a significant amount of micropores and mesopores can be confirmed. Such nanopores exist within the walls of the microhoneycombs. BET surface areas $\left(S_{\mathrm{BET}}\right)$ and mesopore volumes $\left(V_{\text {meso }}\right)$ calculated using the isotherms along with BET parameters, constant $C$ and monolayer coverage $n_{\mathrm{m}}$, are summarized in Table 1 . One unique feature of $\mathrm{SiO}_{2}$ obtained through the ice templating method is that they are microporous. p25 and GRA are nonporous materials, and HSZ is a fairly porous material but its BET surface area and mesopore volume are lower than those of typical $\mathrm{SiO}_{2}$ microhoneycombs. Therefore it is natural that the BET surface areas of the samples including particles will decrease. However, it was confirmed that the BET surface areas and mesopore volumes of the samples were larger than the values calculated by simply adding the values of the $\mathrm{SiO}_{2}$ microhoneycomb and the particles based on their amounts in the microhoneycomb. This indicates that the included particles affect the properties of the $\mathrm{SiO}_{2}$ binder, and nanopores tend to be developed.

As mentioned before, the walls of the honeycombs obtained through the original ice templating method are extremely thin. Accordingly, the diffusion path lengths within such porous walls become extremely short, which is extremely advantageous for porous materials to be used as adsorbents and catalysts. However, the thickness of the walls may also limit the strength of such honeycombs.

In order to verify the effect of particle inclusion on the strength of the microhoneycombs, the compression strengths of the samples were tested using a load cell. The results are also summarized in Table 1. The compression strength increased when HSZ was included, which is thought to be due to the similarity in the composition of $\mathrm{HSZ}$ with the $\mathrm{SiO}_{2}$ matrix. On the other hand, the inclusion of $\mathrm{p} 25$ and GRA led to the decrease in compression strength. However, it was found that the compression strength can be significantly recovered and even improved by calcination, and the strengths of all of the samples easily exceeded the maximum scale of the load cell $\left(3 \mathrm{~kg} / \mathrm{cm}^{2}\right)$.

Finally, the pressure drop which occurs when air was past through the microhoneycombs was measured to evaluate their resistance to flows. The diameter and length of the specimens were $10.5 \mathrm{~mm}$ and $30 \mathrm{~mm}$, 
respectively. A typical result is shown in Fig. 4. As expected, the pressure drop was low even at fairly high flow rates. The pressure drop calculated by assuming that the microhoneycomb can be represented by a bundle of straight identical capillaries is also shown in the figure. The calculated values show the minimum pressure drop values achievable considering the structure, and the actual pressure drop values were only slightly higher then the predicted values. This indicates that there are some irregularities in the channels of the microhoneycomb.

Considering the results obtained through sample characterization, it is assumed that the particles maintain their structure and are dispersed fairly homogeneously throughout the whole $\mathrm{SiO}_{2}$ matrix. Therefore, it can be concluded that microhoneycombs and fibers with unique combined functions can be easily synthesized using the ice templating method.

\section{Conclusion}

In this work, we attempted to widen the range of applicability of the ice templating method by applying this method to mixtures of $\mathrm{SiO}_{2}$ hydrogels and various nanoparticles possessing various functions. It was experimentally shown that microhoneycombs and fibers of $\mathrm{SiO}_{2}$ including various nanoparticles can be synthesized using this modified ice templating method. This indicates that this method can also be applied to materials which cannot be obtained through sol-gel transition, as long as they are combined with sol-gel derivable materials such as a $\mathrm{SiO}_{2}$ gel. It was also found that the morphology which appears during freezing can be controlled by adjusting the firmness of the precursor. From the results of structural characterization, it is assumed that the included particles are uniformly distributed in the $\mathrm{SiO}_{2}$ matrix and that the particles maintain their original structure. We believe this is a promising method to provide materials not only with a unique morphology but also with combined functions.

\section{Acknowledgements}

This work was supported by the Industrial Technology Research Grant Program in 2006, 06B44702a from New Energy and Industrial Technology Development Organization (NEDO) of Japan and the Japan Society for the Promotion of Science (JSPS), Grant-in-Aid for Scientific Research (B), 21360384 (2008).

\section{References}

[1] W. Mahler, M. F. Bechtold, Nature 285 (1980) 27.

[2] T. Kokubo, Y. Teranishi, T. Maki, J. Non-Cryst. Solids 56 (1983) 411.

[3] T. Maki, S. Sakka, J. Mat. Sci. Lett. 5 (1986) 28.

[4] S. R. Mukai, H. Nishihara, H. Tamon, Microporous and Mesoporous Materials 63 (2003) 43.

[5] S. R. Mukai, H. Nishihara, H. Tamon, Chemical Communications (2004) 874.

[6] H. Nishihara, S. R. Mukai, D. Yamashita, H. Tamon, Chemistry of Materials 17 (2005) 683.

[7] S. R. Mukai, H. Nishihara, H. Tamon, Microporous and Mesoporous Materials 116 (2008) 166. 


\section{Figure Captions}

Fig. 1 SEM Images of Typical Samples Obtained in this Work

Fig. 2 XRD Patterns of Typical Microhoneycomb Samples Obtained in this Work

Fig. 3 Nitrogen Adsorption Isotherms of Typical Microhoneycomb Samples Obtained in this Work

Fig. 4 Pressure Drop of a Typical Microhoneycomb Sample

\section{Table Captions}

Table 1 Properties of Typical Microhoneycomb Samples 

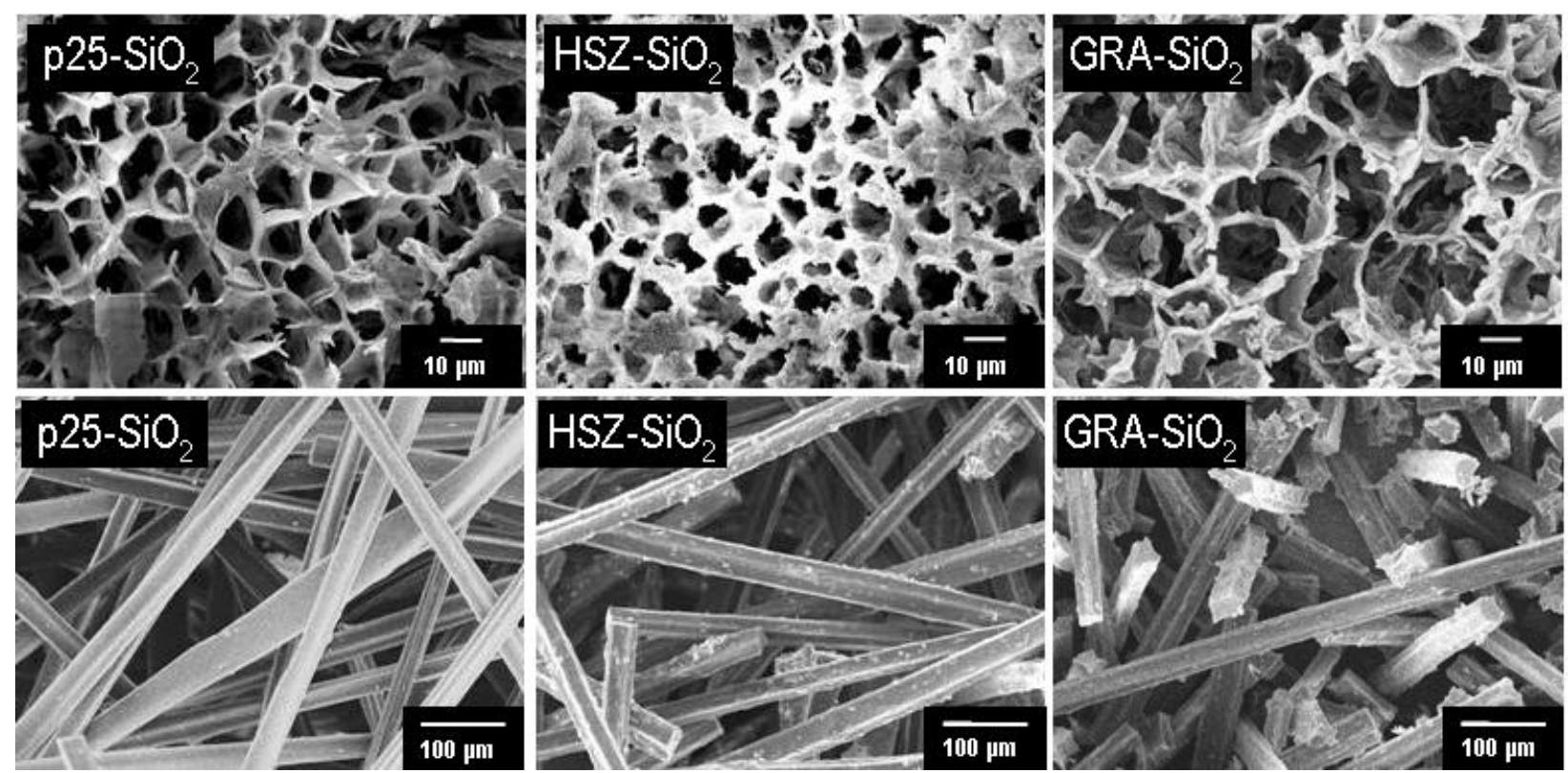

Mukai et al. Fig. 1 

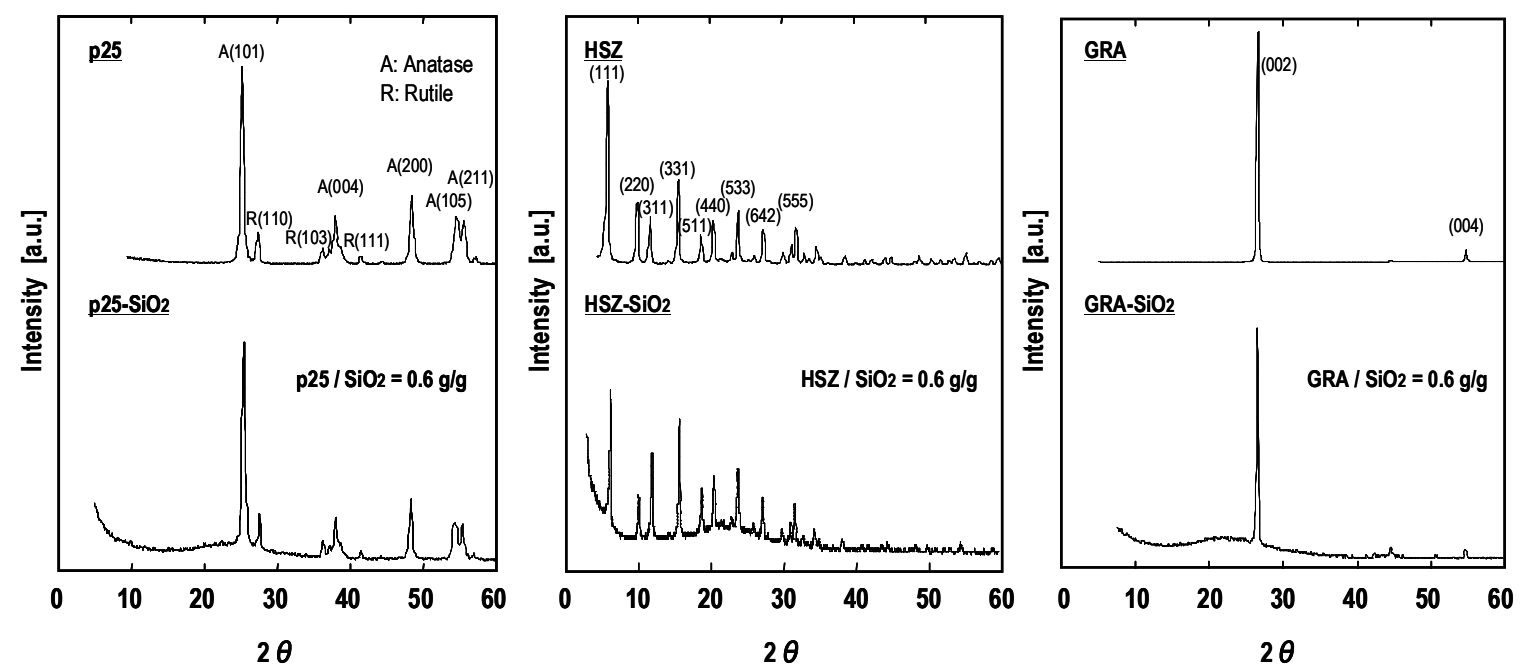

Mukai et al. Fig. 2 


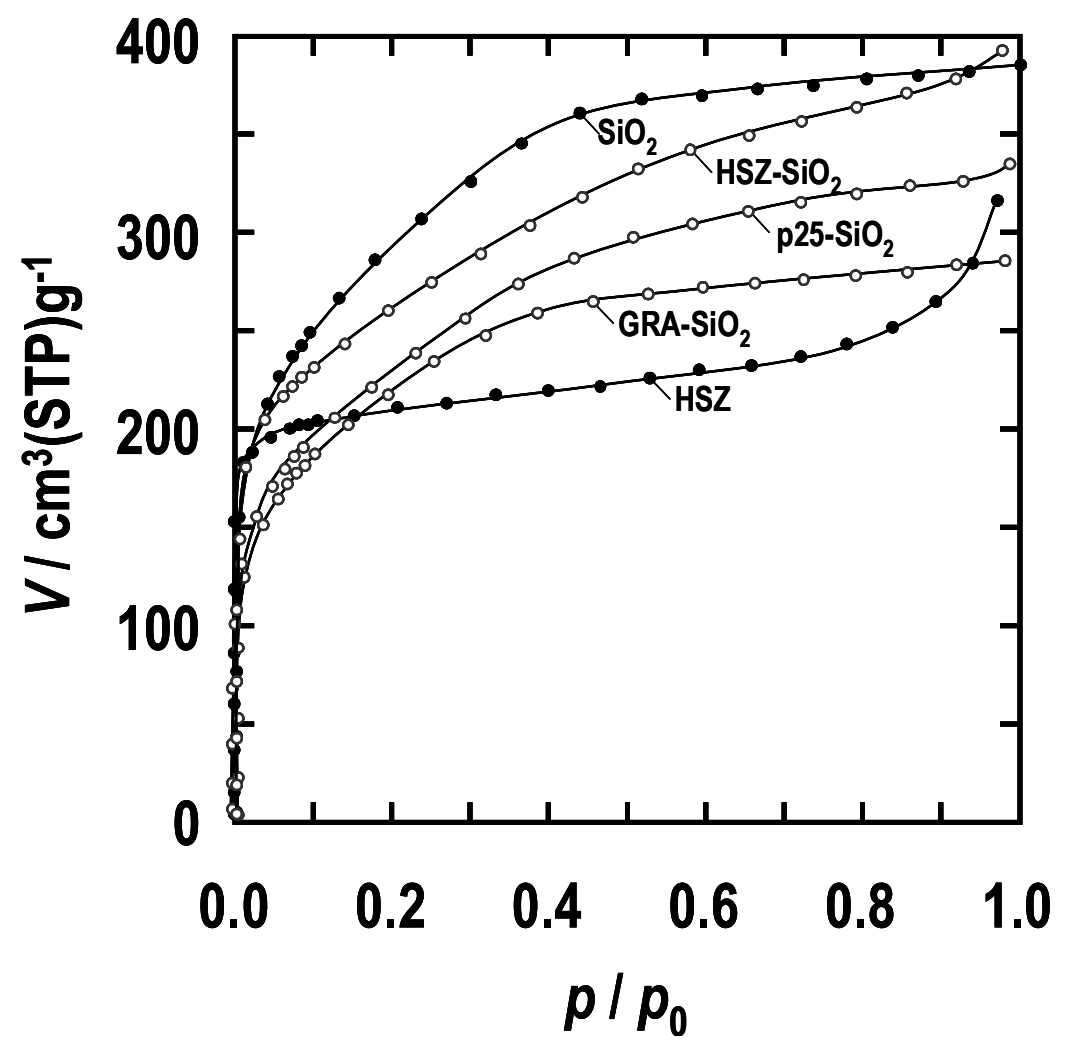

Mukai et al. Fig. 3 


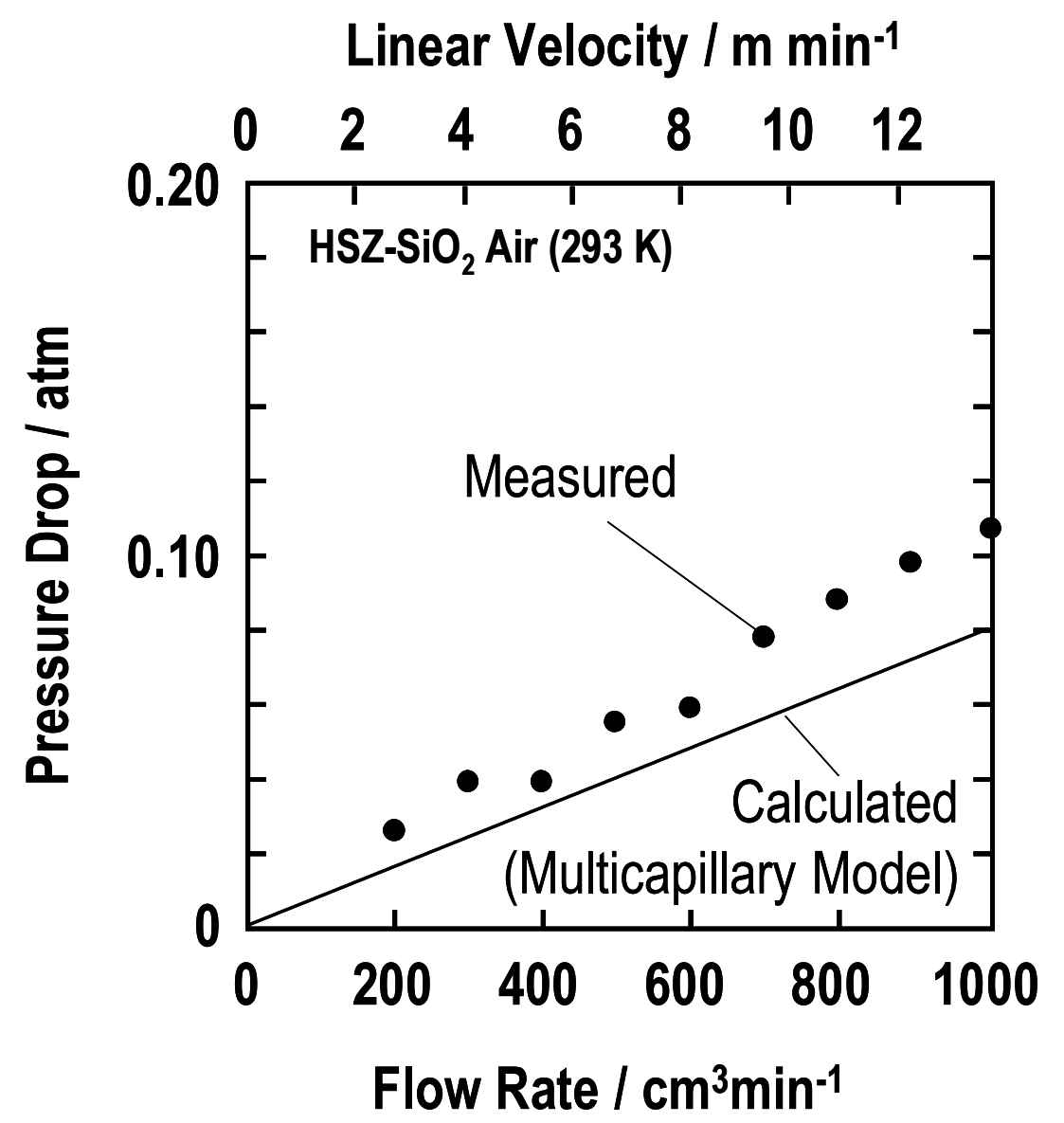

Mukai et al. Fig. 4 


\begin{tabular}{|c|c|c|c|c|c|}
\hline \multirow[t]{2}{*}{ Sample } & \multicolumn{2}{|c|}{ BET Parameters } & \multirow{2}{*}{$\begin{array}{c}S_{\mathrm{BET}} \\
{\left[\mathrm{m}^{2} / \mathrm{g}\right]}\end{array}$} & \multirow{2}{*}{$\begin{array}{c}V_{\text {meso }} \\
{\left[\mathrm{cm}^{3} / \mathrm{g}\right]}\end{array}$} & \multirow{2}{*}{$\begin{array}{c}\text { Compression Strength } \\
{\left[\mathrm{kg} / \mathrm{cm}^{2}\right]}\end{array}$} \\
\hline & $C[-]$ & $n_{m}[\mathrm{~mol} / \mathrm{g}]$ & & & \\
\hline $\mathrm{SiO}_{2}$ Microhoneycomb & 243 & 0.0105 & 1024 & 0.28 & 1.1 \\
\hline $\begin{array}{l}\text { P25-SiO }{ }_{2} \text { Microhoneycomb } \\
\left(\mathrm{p} 25 / \mathrm{SiO}_{2}=0.6 \mathrm{~g} / \mathrm{g}\right)\end{array}$ & 143 & 0.00815 & $795(658)$ & $0.29(0.18)$ & 0.29 \\
\hline $\begin{array}{c}\mathrm{HSZ}^{-\mathrm{SiO}_{2}} \mathrm{Microhoneycomb} \\
\left(\mathrm{HSZ} / \mathrm{SiO}_{2}=0.6 \mathrm{~g} / \mathrm{g}\right)\end{array}$ & 535 & 0.00951 & $927(865)$ & $0.33(0.18)$ & 1.4 \\
\hline $\begin{array}{l}\text { GRA-SiO }_{2} \text { Microhoneycomb } \\
\left(\mathrm{GRA} / \mathrm{SiO}_{2}=0.6 \mathrm{~g} / \mathrm{g}\right)\end{array}$ & 388 & 0.00777 & $757(655)$ & $0.20(0.18)$ & 0.42 \\
\hline
\end{tabular}

*Values in parenthesis are calculated by simply adding the values of the $\mathrm{SiO}_{2}$ microhoneycomb and the particles multiplied by their inclusion ratios 